\title{
The potential dangers of neck manipulation \& risk for dissection and devastating stroke: An illustrative case \& review of the literature
}

\author{
Ryan C Turner ${ }^{1,2,3}$, Brandon P Lucke-Wold ${ }^{1,2,3 *}$, Sohyun Boo ${ }^{4}$, Charles L Rosen ${ }^{1,2,3}$ and Cara L Sedney ${ }^{1}$ \\ ${ }^{1}$ Department of Neurosurgery, West Virginia University School of Medicine, Morgantown, West Virginia \\ ${ }^{2}$ Center for Neuroscience, West Virginia University School of Medicine, Morgantown, West Virginia \\ ${ }^{3}$ West Virginia Clinical \& Translational Science Institute, West Virginia University School of Medicine, Morgantown, West Virginia \\ ${ }^{4}$ Department of Radiology, West Virginia University School of Medicine, Morgantown, West Virginia
}

\begin{abstract}
Chiropractic cervical manipulation is a common practice utilized around the world. Most patients are never cleared medically for manipulation, which can be devastating for those few who are at increased risk for dissections. The high velocity thrust used in cervical manipulation can produce significant strain on carotid and vertebral vessels. Once a dissection has occurred, the risk of thrombus formation, ischemic stroke, paralysis, and even death is drastically increased. In this case report, we highlight a case of a 32-year-old woman who underwent chiropractic manipulation and had vertebral artery dissection with subsequent brainstem infarct. She quickly deteriorated and passed away shortly after arrival to the hospital. Although rare, one in 48 chiropractors have experienced such an event. We utilize this case to highlight the risk associated with cervical manipulation and urge open dialogue between chiropractors and physicians. Receiving medical clearance prior to cervical manipulation in potential at risk patients would drastically reduce morbidity and mortality.
\end{abstract}

\section{Introduction}

Spinal manipulation, a hallmark practice of chiropractic providers and a practice occasionally utilized by physiotherapists, osteopaths, and physicians, is often viewed as a controversial and potentially dangerous procedure without good evidence supporting its use [1]. Specifically, epidemiologic associations have been observed between chiropractic maneuvers and cervical artery dissection, although it has been difficult to prove causal relationship [2-4]. The potential consequence of cervical artery dissection is stroke, which may have a range of consequences with regards to long-term morbidity and mortality [5-7].

Herein, we report a case of vertebral artery dissection and subsequent brainstem infarction immediately following chiropractic manipulation in a young female patient. This patient experienced rapid deterioration immediately following manipulation and proceeded to undergo cardiac arrest prior to presentation to the hospital and subsequent death. This case serves to highlight the potential dangers of chiropractic manipulation and provides a thorough review of the literature relating chiropractic manipulation and stroke. Furthermore, this case presentation demonstrates a likely causal relationship although prior imaging was not available to rule out a pre-existing dissection. The case therefore satisfies many of the criteria proposed by Hill [8] and used by Tuchin [9] to refute the role of chiropractic manipulation in dissection and stroke.

\section{Case Presentation}

The patient, a 32-year-old Caucasian woman, presented to the West Virginia University Hospital Emergency Department via Emergency Medical Services. The patient had been at her usual baseline state of health with no significant past medical history prior to visiting the chiropractor for neck adjustment earlier that day for tension like soreness. The patient underwent neck manipulation after which she immediately complained of neck pain, diaphoresis, and proceeded to experience cardiac and respiratory arrest. Emergency Medical Services was called, and cardiopulmonary resuscitation was performed with one round of epinephrine administered. It was reported that the patient was pulseless and apneic for 3 minutes prior to EMS arrival. The patient was intubated on transport and her Glasgow Coma Scale score was 3T prior to arrival. Mean arterial blood pressure was 80 with palpable femoral pulses at arrival to the emergency department. Upon arrival in the emergency department, a CT stroke protocol was performed which demonstrated bilateral severe distal cervical vertebral artery dissections with acute thrombotic emboli seen in the left cervical vertebral artery (Figures 1 and 2). This was accompanied by complete occlusion of the basilar tip including the proximal posterior cervical arteries. The patient received an initial bolus of intravenous tissue plasminogen activator (IV rtPA) at this time and the decision was made to proceed with endovascular intervention given the recent onset of occlusion.

The patient was brought to the neurovascular angiography suite and femoral access obtained. Angiography of the left vertebral artery

Correspondence to: Brandon Lucke-Wold, Department of Neurosurgery, West Virginia University School of Medicine, Morgantown, West Virginia, E-mail: Bwold@mix.wvu.edu

Key words: Chiropractic manipulation, Vertebral artery dissection, Brainstem infarct, Open communication, Medical clearance

Received: March 08, 2018; Accepted: March 22, 2018; Published: March 25, 2018 


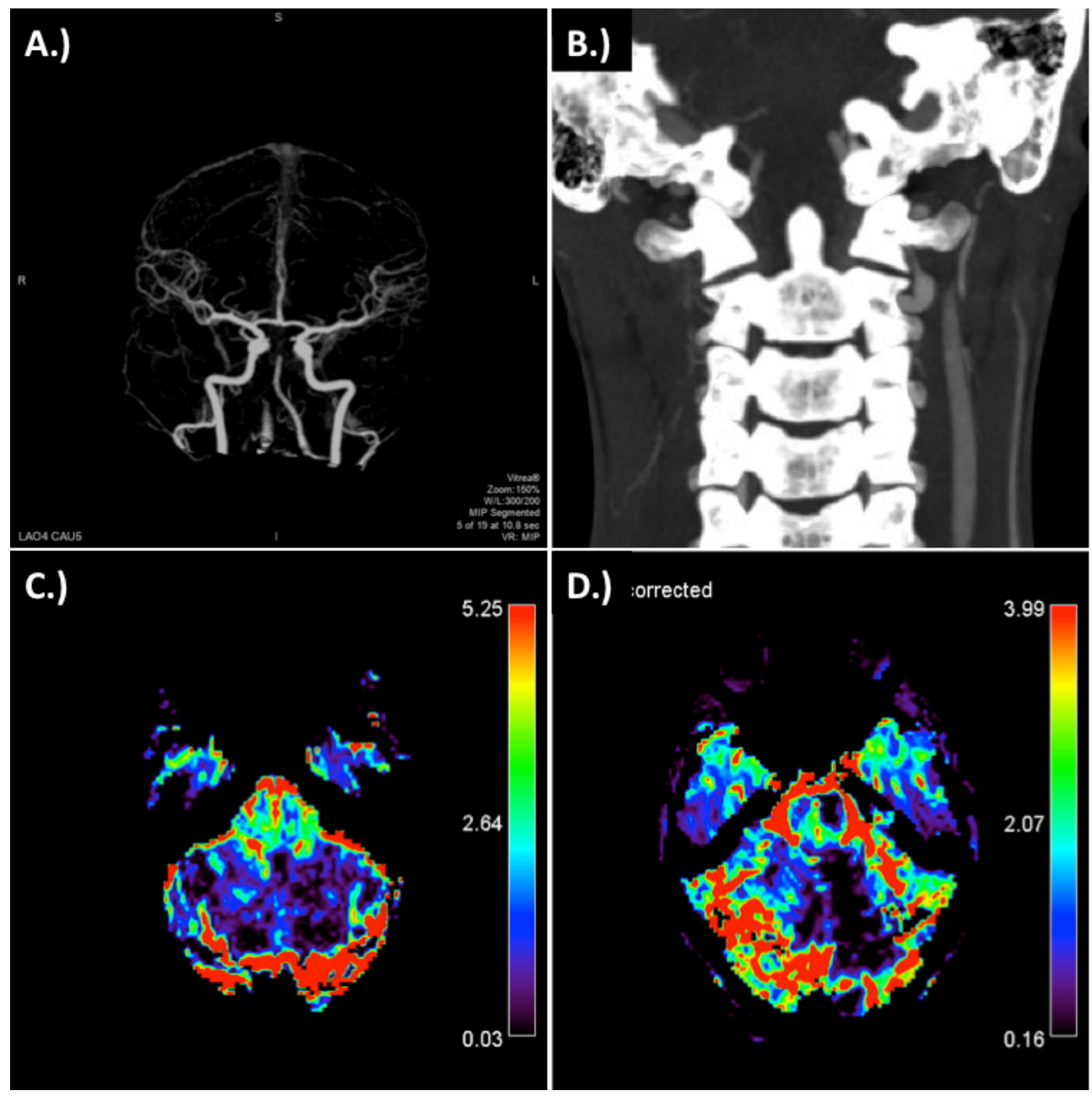

Figure 1. CT Stroke Protocol at time of presentation. A.) 4D CTA demonstrating top of the basilar occusion and poor flow in the right vertebral artery; B.) coronal CA demonstrating dissection of verterbral arteries at the C1-C2 level, particularly prominent on the right; C.) relative blood volume; and D.) corrected relative blood volume.

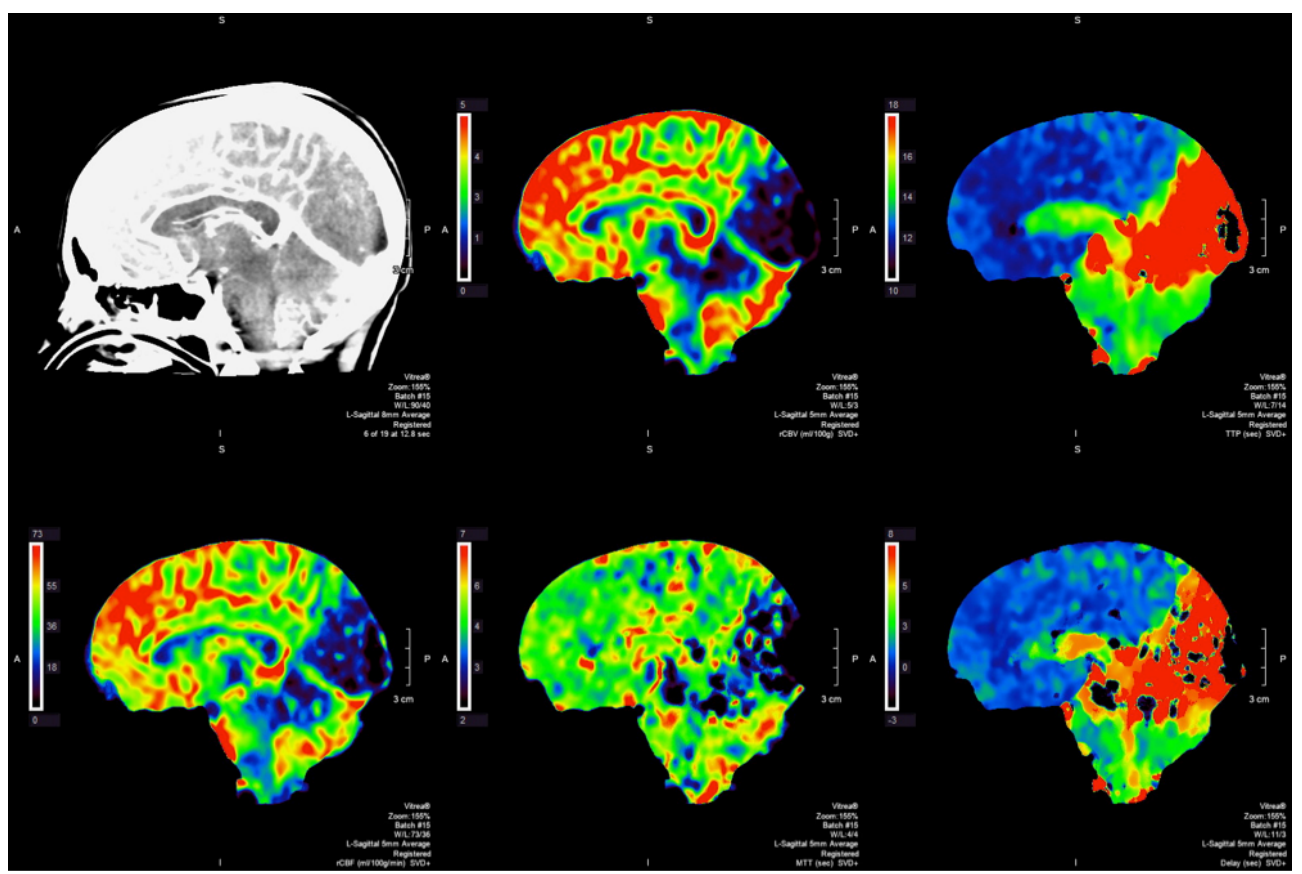

Figure 2. CT Stroke Protocol with perfusion imaging demonstrating primary involvement of occipital lobe, cerebellum, and brainstem. Perfusion imaging reflecting rCBV, TTP, rCBF, MTT, and delay (from top-left to bottom-right). 
demonstrated severe dissection involving the distal cervical vertebral artery segments at the C1-C2 level with presence of sub occlusive thrombi. There was an occlusive clot in the left Posterior Inferior Cerebellar Artery (PICA). Intracranial imaging demonstrated occlusion at the basilar apex with absent filling into the right Posterior Cerebral Artery (PCA). There was occlusion of the distal left PCA. Angiography of the right vertebral artery demonstrated severe dissection of the distal cervical vertebral artery at C1-C2 with the presence of trickle-like flow into the vertebrobasilar junction. No filling was observed in the right PICA territory (Figure 3). At this point, it was decided that the left vertebral artery offered the best access to the basilar trunk.

Subsequently, distal aspiration was begun with a Penumbra 5 Max ACE distal aspiration catheter which initially demonstrated slow flow through the suction tubing. The 5 Max ACE was withdrawn into the proximal basilar artery until flow was seen within the suction tubing. Repeat angiography at this time demonstrated recanalization of the basilar apex and proximal PCAs. TICI3 perfusion was seen in the right PCA. Occlusive clot remained in the left distal P2 segment. Given the large size of the PCA, timing of events, and patient's age, the decision was made to attempt clot retrieval of this. At this time, the Trevo ProVue microcatheter was navigated into the left distal PCA distal to the clot. The Trevo $4 \mathrm{~mm}$ x $30 \mathrm{~mm}$ stent retriever was deployed for approximately 3 minutes. The suction canister was attached, and the stent retriever was pulled with distal aspiration. No significant recanalization was achieved with what amounted to TICI0 perfusion to the left PCA territory. No further attempts were made as it was believed that the left PCA territory had completed its infarction.
Following completion of endovascular therapy, the patient was taken for immediate MRI Brain with and without contrast for assessment of brainstem integrity and cerebrovascular status prior to transport to the intensive care unit. MRI demonstrated extensive areas of restricted diffusion accompanied by perfusion abnormalities consistent with acute infarction of the posterior circulation, specifically within the bilateral cerebellar hemispheres, right medulla, pons bilaterally, midbrain, thalami, and left occipital lobe (Figure 4). The following day, additional CT Brain imaging was acquired and demonstrated signs of elevation of intracranial pressure with hydrocephalus, worsening of cerebral edema diffusely, hemorrhagic transformation of the left occipital lobe, continued infarct evolution within the posterior circulation, and cerebellar tonsillar herniation.

\section{Discussion}

Arterial dissection, particularly as related to the cervical arteries and risk of ischemic stroke, has become a topic of increasing interest in recent years. Many potential risk factors have been identified that include genetic disorders such as $\alpha_{1}$-antitrypsin ( $\alpha 1-\mathrm{AT}$ ) deficiency [10-12], connective tissue disease [13-15], MTHFR TT genotype [16], homocysteine concentration within the serum [16,17], history of migraine [18-20], variations in cardiac/cervical vessel anatomy [21-25], infection $[10,19]$, oral contraceptive use $[10,18,20]$, and trivial trauma [26-30]. A more rigorous analysis of these studies in the systematic review by Rubinstein and colleagues and additional completion of larger/more focused studies have failed to confirm that many of these variables are risk factors for dissection [31].
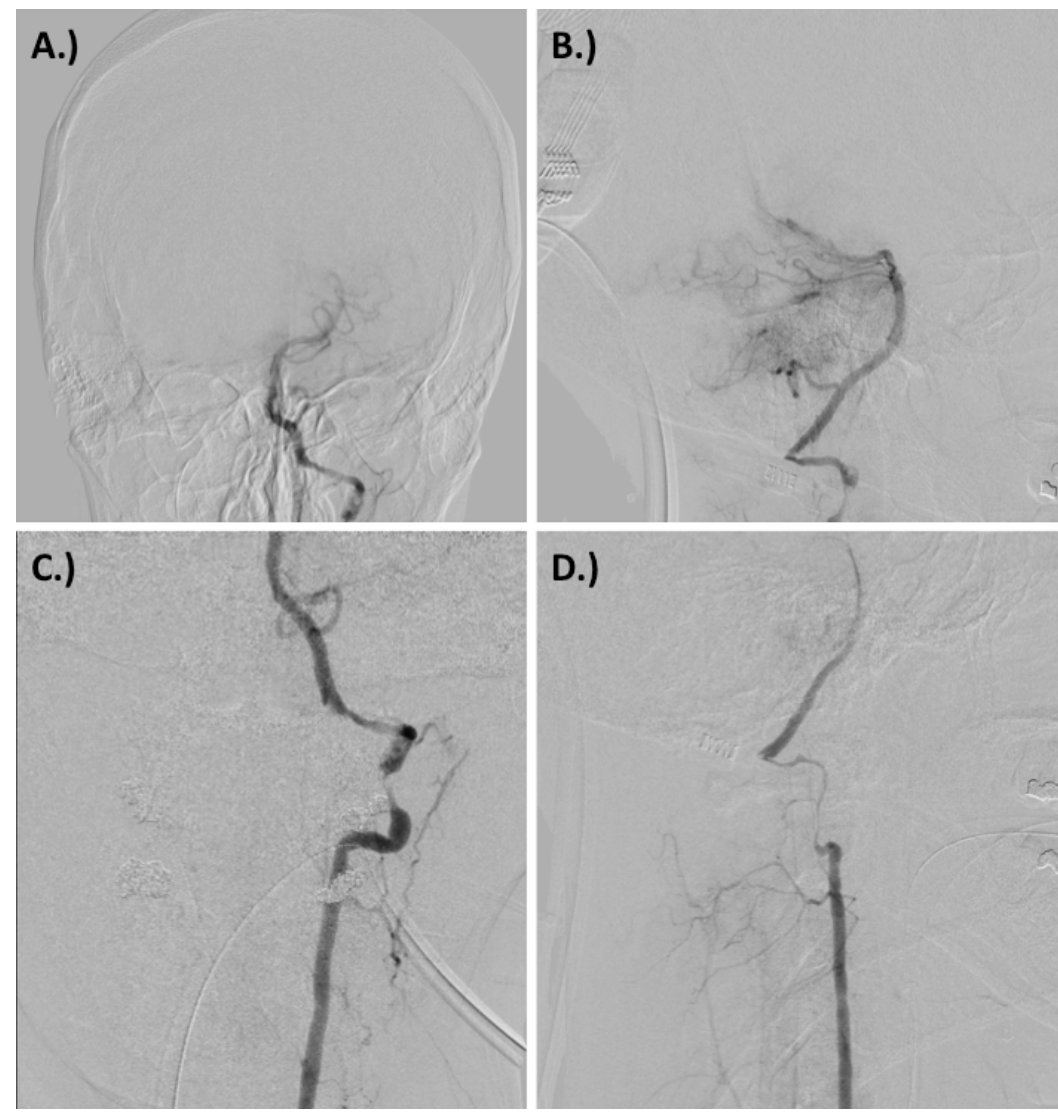

Figure 3. Digital subtraction angiography at time of presentation demonstrating A.) anterior-posterior view showing basilar and PICA occlusion, B.) lateral view showing basilar and PICA occlusion, C.) left vertebral artery dissection, and D.) right vertebral artery dissection. 

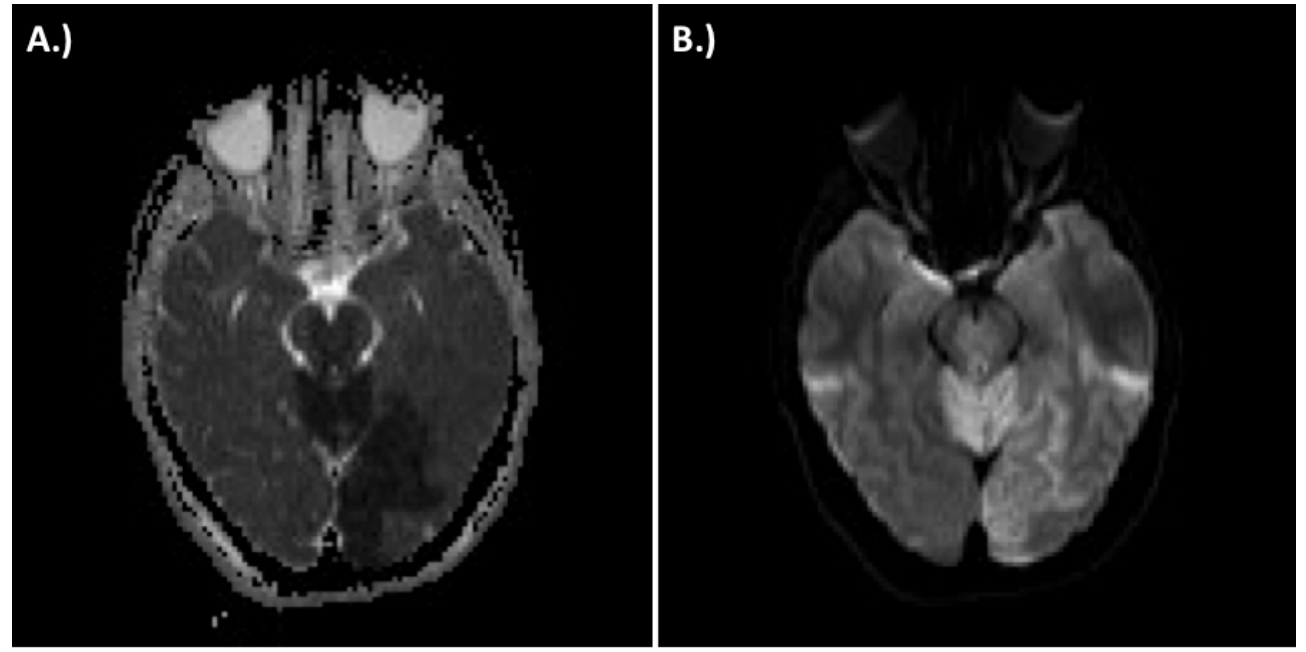

Figure 4. Immediate post-procedure magnetic resonance imaging demonstrating ischemic stroke within the cerebellum, brainstem, and left occipital cortex. A.) attenuated diffusion coefficient (ADC) sequence capturing left occipital stroke and B.) diffusion-weighted imaging capturing cerebellar, brainstem, and left occipital cortex stroke.

Cerebral and vertebral artery dissections can occur both at the chiropractor's office and at home when practicing self-manipulation techniques [32]. Multi-artery dissection is a rare occurrence following chiropractic manipulation but can lead to extensive infarct and adverse outcomes [33]. A recent systematic review found that 901 cases of cerebral artery dissections have been reported in the literature in relation to chiropractic manipulation. In 707 of these cases, the patient went on to develop some type of stroke [34]. Notably, a study focused on malpractice data from the Canadian Chiropractic Protective Association (CCPA) showed a low incidence of neurological symptoms attributable to cervical manipulation and arterial dissection following chiropractic manipulation [35]. Specifically, data from the report suggests that a chiropractor will be aware of an arterial dissection occurring following cervical manipulation only once in 8.06 million office visits with only one in forty-eight chiropractors experiencing such an event in their entire careers [35]. Chiropractors are inexperienced in detecting the signs and symptoms of a dissection.

Diagnosing thrombosis early in these cases is critical for successful endovascular or surgical intervention [36]. For example, a recent case report showed that a patient with vertebral artery dissection and infarction following chiropractic manipulation was successfully treated with mannitol and ventriculostomy [37]. Unfortunately, despite early identification, cerebrovascular injury has resulted in at least 26 reported deaths following chiropractic spinal manipulation [38]. For our patient, clot formation was observed in the PICA, basilar apex, and distal P2 segment limiting endovascular and surgical options. Endovascular diagnosis and attempted treatment failed. The patient subsequently went on to develop hemorrhagic transformation, severe cerebral edema, and ultimately cerebellar tonsillar herniation. The key learning points for this case revolve around prevention.

Futch et al., 2015 urge chiropractic colleagues to have a high suspicion of vertebral artery dissection when patients come in for treatment with sudden neck pain and headache [39]. The use of magnetic resonance angiography in these cases may prevent the chiropractor from performing potentially dangerous manipulations in at risk individuals. Green and colleagues present modified manipulation techniques that don't involve high velocity movements. These techniques might minimize the rotation of the neck and can potentially prevent vascular dissections [40]. In a cadaveric study, Piper and colleagues show that strain and peak angular displacement during spinal manipulation is highly variable between individuals. The peak strain often occurs at an intermediate point during the movement of the neck [41]. Therefore, even in experienced hands, a high velocity spinal manipulation may not be safe based on individual anatomical differences in patients

Based on the several reported cases of complications following spinal manipulation, we encourage increased communication between chiropractic and medical colleagues. A low threshold should exist for chiropractors to consult physicians in order to make sure the patient is cleared for manipulation. If the patient has been seen at a medical facility and MRI or CT images are suggestive of potential risks, this information needs to be communicated to the patient regarding heightened potential for adverse outcomes related to manipulation. Tarola and Phillips report a case where acute onset neck pain in a patient motivated the chiropractor to refer the patient to the emergency department before manipulation. MRA revealed vertebral artery dissection therefore preventing a potentially devastating outcome if manipulation had been performed [42]. In the future, mechanoreceptor data may be a viable option to investigate the safety of manipulation and thrust procedures on muscle spindle responses [43]. This neural based stimulation in conjunction with clinical judgment may be used to select patients at low risk for vascular injury.

\section{Conclusion}

High velocity thrust manipulation of the cervical spine places the carotid and vertebral arteries at risk of dissection. Even with early diagnosis, thrombus occlusion and subsequent infarction can lead to serious adverse outcomes. We report a case of a young woman who had bilateral vertebral artery dissections following chiropractic manipulation. The patient had thrombus occlusion that was not amenable to endovascular treatment and developed hemorrhagic transformation, cerebral edema, and cerebellar herniation. Additionally, ongoing research is necessary to determine the safety of cervical manipulation and whether the techniques should be modified in order to prevent injury.

\section{References}

1. Ernst E (2007) Adverse effects of spinal manipulation: a systematic review. $J R$ Soc Med 100: 330-338. [Crossref]

2. Preul C, Joachimski F, Witte OW, Isenmann S (2010) Bilateral vertebral artery dissection after chiropractic maneuver. Clin Neuroradiol 20: 255-259. [Crossref] 
3. Reuter U, Hamling M, Kavuk I, Einhaupl KM, Schielke E (2006) Vertebral artery dissections after chiropractic neck manipulation in Germany over three years. $J$ Neurol 253: 724-730. [Crossref]

4. Thanvi B, Munshi SK, Dawson SL, Robinson TG (2005) Carotid and vertebral artery dissection syndromes. Postgrad Med J 81: 383-388. [Crossref]

5. Biller J, Sacco RL, Albuquerque FC, Demaerschalk BM, Fayad P, et al. (2014) Cervical arterial dissections and association with cervical manipulative therapy: a statement for healthcare professionals from the american heart association/american stroke association. Stroke 45: 3155-3174. [Crossref]

6. Cassidy JD, Boyle E, Cote P, He Y, Hogg-Johnson S, et al. (2008) Risk of vertebrobasilar stroke and chiropractic care: results of a population-based case-control and case-crossover study. Spine (Phila Pa 1976) 33: S176-183. [Crossref]

7. Rothwell DM, Bondy SJ, Williams JI (2001) Chiropractic manipulation and stroke: a population-based case-control study. Stroke 32: 1054-1060. [Crossref]

8. Hill AB (1965) The Environment and Disease: Association or Causation? Proc R Soc Med 58: 295-300. [Crossref]

9. Tuchin P (2013) Chiropractic and stroke: association or causation? Int J Clin Pract 67: 825-833. [Crossref]

10. Grau AJ, Brandt T, Buggle F, Orberk E, Mytilineos J, et al. (1999) Association of cervical artery dissection with recent infection. Arch Neurol 56: 851-856. [Crossref]

11. Konrad C, Langer C, Muller GA, Berger K, Dziewas R, et al. (2005) Protease inhibitors in spontaneous cervical artery dissections. Stroke 36: 9-13.

12. Vila N, Millan M, Ferrer X, Riutort N, Escudero D (2003) Levels of alpha1-antitrypsin in plasma and risk of spontaneous cervical artery dissections: a case-control study. Stroke 34: E168-169. [Crossref]

13. Brandt T, Hausser I, Orberk E, Grau A, Hartschuh W, et al. (1998) Ultrastructural connective tissue abnormalities in patients with spontaneous cervicocerebral artery dissections. Ann Neurol 44: 281-285. [Crossref]

14. Brandt T, Orberk E, Weber R, Werner I, Busse O, et al. (2001) Pathogenesis of cervical artery dissections: association with connective tissue abnormalities. Neurology 57: 2430. [Crossref]

15. Hausser I, Muller U, Engelter S, Lyrer P, Pezzini A, et al. (2004) Different types of connective tissue alterations associated with cervical artery dissections. Acto Neuropathol 107: 509-514. [Crossref]

16. Pezzini A, Del Zotto E, Archetti S, Negrini R, Bani P, et al. (2002) Plasma homocysteine concentration, C677T MTHFR genotype, and 844ins68bp CBS genotype in young adults with spontaneous cervical artery dissection and atherothrombotic stroke. Stroke 33: 664-669. [Crossref]

17. Konrad C, Muller GA, Langer C, Kuhlenbaumer G, Berger K, et al. (2004) Plasma homocysteine, MTHFR C677T, CBS 844ins68bp, and MTHFD1 G1958A polymorphisms in spontaneous cervical artery dissections. J Neurol 251: 1242-1248. [Crossref]

18. D'Anglejan-Chatillon J, Ribeiro V, Mas JL, Youl BD, Bousser MG (1989) Migraine--a risk factor for dissection of cervical arteries. Headache 29: 560-561. [Crossref]

19. Guillon B, Berthet K, Benslamia L, Bertrand M, Bousser MG, et al. (2003) Infection and the risk of spontaneous cervical artery dissection: a case-control study. Stroke 34 e79-81. [Crossref]

20. Tzourio C, Benslamia L, Guillon B, Aidi S, Bertrand M, et al. (2002) Migraine and the risk of cervical artery dissection: a case-control study. Neurology 59: 435-437. [Crossref]

21. Barbour PJ, Castaldo JE, Rae-Grant AD, Gee W, Reed JF 3rd, et al. (1994) Internal carotid artery redundancy is significantly associated with dissection. Stroke 25: 12011206. [Crossref]

22. Calvet D, Boutouyrie P, Touze E, Laloux B, Mas JL, et al. (2004) Increased stiffness of the carotid wall material in patients with spontaneous cervical artery dissection. Stroke 35: 2078-2082. [Crossref]

23. Guillon B, Tzourio C, Biousse V, Adraï V, Bousser MG, et al. (2000) Arterial wall properties in carotid artery dissection: an ultrasound study. Neurology 55: 663-666. [Crossref]
24. Lucas C, Lecroart JL, Gautier C, Leclerc X, Dauzat M, et al. (2004) Impairment of endothelial function in patients with spontaneous cervical artery dissection: evidence for a general arterial wall disease. Cerebrovasc Dis 17: 170-174. [Crossref]

25. Tzourio C, Cohen A, Lamisse N, Biousse V, Bousser MG (1997) Aortic root dilatation in patients with spontaneous cervical artery dissection. Circulation 95: 2351-2353. [Crossref]

26. d'Anglejan Chatillon J, Ribeiro V, Mas JL, Bousser MG, Laplane D (1990) Dissection of the extracranial internal carotid artery. 62 cases. Presse Med 19: 661-667. [Crossref]

27. Desfontaines P, Despland PA (1995) Dissection of the internal carotid artery: aetiology, symptomatology, clinical and neurosonological follow-up, and treatment in 60 consecutive cases. Acta Neurol Belg 95: 226-234. [Crossref]

28. Dziewas R, Konrad C, Drager B, Evers S, Besselmann M, et al. (2003) Cervical artery dissection--clinical features, risk factors, therapy and outcome in 126 patients. $J$ Neurol 250: 1179-1184. [Crossref]

29. Leys D, Moulin T, Stojokovic T, Begey S, Chavot D (1995) DONALD Investigators Follow-up of patients with history of cervical artery dissection. Cerebrovasc Dis 5 : 43-49.

30. Touze E, Gauvrit JY, Moulin T, Meder JF, Bracard S, et al. (2003) Risk of stroke and recurrent dissection after a cervical artery dissection: a multicenter study. Neurology 61: 1347-1351. [Crossref]

31. Rubinstein SM, Peerdeman SM, van Tulder MW, Riphagen I, Haldeman S (2005) A systematic review of the risk factors for cervical artery dissection. Stroke 36: 15751580. [Crossref]

32. Mosby JS, Duray SM (2011) Vertebral artery dissection in a patient practicing selfmanipulation of the neck. J Chiropr Med 10: 283-287. [Crossref]

33. Melikyan G, Kamran S, Akhtar N, Deleu D, Miyares FR (2015) Cortex-sparing infarction in triple cervical artery dissection following chiropractic neck manipulation. Qatar Med J 16. [Crossref]

34. Wynd S, Westaway M, Vohra S, Kawchuk G (2013) The quality of reports on cervical arterial dissection following cervical spinal manipulation. PloS one 8: e59170. [Crossref]

35. Haldeman S, Carey P, Townsend M, Papadopoulos C (2001) Arterial dissections following cervical manipulation: the chiropractic experience. CMAJ 165: 905-906. [Crossref]

36. Mikkelsen R, Dalby RB, Hjort N, Simonsen CZ, Karabegovic S (2015) Endovascular Treatment of Basilar Artery Thrombosis Secondary to Bilateral Vertebral Artery Dissection with Symptom Onset Following Cervical Spine Manipulation Therapy. Am J Case Rep 16: 868-871. [Crossref]

37. Jones J, Jones C, Nugent K (2015) Vertebral artery dissection after a chiropractor neck manipulation. Proc (Bayl Univ Med Cent) 28: 88-90. [Crossref]

38. Ernst E (2010) Deaths after chiropractic: a review of published cases. Int J Clin Pract 64: 1162-1165. [Crossref]

39. Futch D, Schneider MJ, Murphy D, Grayev A (2015) Vertebral artery dissection in evolution found during chiropractic examination. BMJ Case Rep. [Crossref]

40. Green BN, Browske LK, Rosenthal CM (2014) Elongated styloid processes and calcified stylohyoid ligaments in a patient with neck pain: implications for manual therapy practice. J Chiropr Med 13: 128-133. [Crossref]

41. Piper SL, Howarth SJ, Triano J, andHerzog W (2014) Quantifying strain in the vertebral artery with simultaneous motion analysis of the head and neck: a preliminary investigation. Clin Biomech (Bristol, Avon) 29: 1099-1107. [Crossref]

42. Tarola G, Phillips RB2 (2015) Chiropractic Response to a Spontaneous Vertebral Artery Dissection. J Chiropr Med 14: 183-190. [Crossref]

43. Reed WR, Liebschner MA, Sozio RS, Pickar JG, Gudavalli MR (2015) Neural Response During a Mechanically Assisted Spinal Manipulation in an Animal Model: A Pilot Study. J Nov Physiother Phys Rehabil 2: 20-27. [Crossref]

Copyright: (C2018 Turner RC. This is an open-access article distributed under the terms of the Creative Commons Attribution License, which permits unrestricted use, distribution, and reproduction in any medium, provided the original author and source are credited. 